\title{
Reconversión productiva y capacitación del personal en el sector lácteo en Argentina
}

\author{
Pujol, Andrea
}

\section{Resumen}

El artículo presenta algunos desarrollos y conclusiones acerca de la vinculación existente entre los desafíos comerciales y productivos que enfrentan las empresas y las políticas y acciones de capacitación que implementan. La información surge de un estudio descriptivo, de corte cualitativo, sobre siete empresas del sector lácteo argentino, que incluyó un estudio en profundidad de tres de las unidades productivas observadas. El propósito del estudio consistió en analizar las características que adquiere la capacitación del personai en tales empresas. En el desarrollo se pudo constatar que la implementación de sistemas de calidad y la innovación tecnológica constituyen los principales factores impulsores de acciones de capacitación, aún cuando no siempre derivan en el desarrollo de politicas a largo plazo. En algunos procesos de reconversión la capacitación se instala como valor de la cultura y en otros solo adquiere un carácter instrumental y contingente, llegando a considerársele más como un costo que como una inversión. La gestión de las innovaciones produce a su vez un impacto importante en la división social del trabajo y en la composición demográfica de las empresas, dando origen a variaciones significativas en cuanto a la edad, el nivel educativo de ingreso y la calificación del personal.

Palabras clave: Reconversión productiva, innovación tecnológica, capacitación, sector lácteo, Argentina.

\section{Productive Reconversion and Manpower Retraining in the Dairy Sector in Argentina}

\section{Abstract}

The article presents some developments and conclusions about the link between productive and commercial challenges faced by firms and the re-training actions and policies they implement.

\section{Recibido: 00-09-27, Aceptado: 00-11-06}

Investigador Jefe del Area de Recursos Humanos del Instituto de Administración de la Facultad de Ciencias Económicas. Universidad Nacional de Córdoba. Argentina.

E-Mall: apujol@eco.uncor.edu. Web: http://eco.uncor.edu/organización/institutos/institutos-in.htm 
The information comes from a qualitative and descriptive survey of 7 (seven) Argentine firms in the diary sector, which included a detailed study of 3 (three) of the observed productive units. The purpose of this study was to analyze the characteristics that retraining of personnel assume in firms belonging to the Argentine dairy sector. On carrying out the study, the results confirmed that the implementation of quality control systems and technological innovations are the main factors setting off retraining actions, even though they do not always turn out to be long-term policies. In some re-conversion processes, retraining takes on cultural values, while in others it has a more contingent and instrumental feature. In some cases being it is considered to be more of a cost than an investment. Innovation management causes, in turn, an important impact on the social division of labor and on the demographic composition of firms, giving way to significant variations in age, income, educational level, and manpower skills.

Key words: Productive reconversion, technological innovation, retraining, dairy sector, Argentina.

\section{Introducción}

El propósito del presente escrito consiste en comunicar algunas conclusiones parciales y reflexiones surgidas de la información obtenida en un trabajo de investigación sobre siete empresas del sector lácteo argentino'. El propósito fue indagar la importancia que conceden las empresas a la capacitación del personal, qué estrategias despliegan en este sentido, bajo qué condiciones organizacionales se desarrollan y cuáles son los alcances de los resultados de su implementación.
El desarrollo del estudio ${ }^{2}$ permitió analizar la vinculación existente entre los desafíos comerciales y productivos que enfrentan las empresas y las políticas y acciones de capacitación que implementan. En este marco, resulta factible enunciar algunas proposiciones e interrogantes acerca de la naturaleza de los condicionantes que adquieren los procesos de reconversión de competencias del personal en las empresas observadas.

En la primera parte del presente escrito se describe el contexto teórico de la problemática y posteriormente, se recons-

1 Se trata un estudio desarrollado desde el Instituto de Administración de la Facultad de Ciencias Económicas de la Universidad Nacional de Córdova entre 1996 y 1999: "Los desafíos de la reconversión laboral: la capacitaçión en las empresas del sector lácteo".

2 Desde el punto de vista metodológico, las principales actividades que articularon el proceso de investigación fueron: a) análisis de documentación económica del sector con el fin de extraer datos de su dinámica económica y empresarial; b) contactos significativos con instituciones formadoras, entidades sindicales y con la cámara industrial que agrupa el sector, c) visitas, entrevistas y observaciones en varias fábricas, con el objeto de realizar una aproximación a las características generales de la estructura organizativa de la industria láctea, conocer el proceso de producción de algunos productos y su tecnología característica; d) aplicación de un cuadernillo de relevamiento de información en siete empresas con la finalidad de recoger información general sobre las mismas, la gestión de recursos humanos y la oferta de capacitación; e) estudio en profundidad de tres casos, con el fin de ganar terreno en profundidad de análisis, que in- 
truyen algunas prácticas observadas en las empresas recuperando la voz de los actores sociales, con el fin de sistematizar algunas inferencias significativas.

\section{Desarrollo}

\subsection{El problema y su contexto teórico}

La estrecha vinculación entre reconversión de la empresa y reconversión laboral es destacada actuaimente por la mayoría de los estudios del trabajo que se realizan en Latinoamérica (Novick, 1999; Labarca, 1999).

En la explicación de los procesos de cambio, se destaca la centralidad que adquiere el trabajo humano en el enfoque de la teoria de la regulación (Aglietta, 1979; Lipietz, 1985; Boyer, 1989). Esta perspectiva permite comprender las formas institucionales y de organización que predominan en la actualidad en función de las crisis que sufren los distintos modelos de división social y técnica del tra bajo, desde el análisis de la crisis endógena del taylorismo y el fordismo. A su vez, los estudios desarrollados sobre la evolución de los procesos de trabajo han permitido elucidar el impacto de la emergencia de un nuevo paradigma productivo "flexible" en las relaciones salariales y en la organización del trabajo.

Bajo la noción de sistema productivo flexible (Coriat, 1992; Neffa, 1998), las nuevas formas de organización del trabajo (NFOT) y las nuevas tecnologías (NT) representan hoy el elemento central de la transformación del mundo del trabajo e implican importantes cambios a nivel de la gestión estratégica, financiera y comercial, de la gestión productiva y de la gestión de recursos humanos de la empresa.

Las principales características productivas del modelo de producción flexible son: a) modificación de las estructuras organizativas: menos niveles jerárquicos y reducción del tamaño de las organizaciones a través de la descentrallzación y la desconcentración de actividades; b) incorporación de nuevas tecnologías materiales para la automatización de procesos e informatización de las comunicaciones e innovación en las tecnologías de gestión o tecnologías sociales, con predominio del trabajo en equipo, en células o en redes; c) producción en series pequeñas de productos heterogéneos, que se realizan una vez que los productos ya han sido vendidos, reduciéndose los costos de capital y el costo laboral por la disminución de stocks (just in time) y d) producción integrada con escasa división de tareas y mayor participación funcional de los trabajadores en la concepción y programación de las actividades, lo que disminuye las actividades repetitivas, monótonas y poco creativas (Stankiewicz, 1991).

La modificación sustantiva de la arquitectura organizacional consiste en

cluyo observaciones sistemáticas, entrevistas en profundidad a gerentes, jefes, supervisores y operarios, análisis de documentación institucional-folletos, carpeta de presentación de la empresa, etc.,- y de documentación interna - organigramas, reglamentos, lay outs de planta, planes de capacitación, etc. 
Reconversión productiva y capacitación del personal en el sector lácteo en Argentina Pujol, Andrea

parte en un acortamiento de distancias significativo entre "los que piensan" y "los que hacen", trasladándose muchas funciones de los cuadros medios y altos a los niveles operativos. En muchos casos, los niveles medios que antes eran esenciales para servir de puente entre el nivel operativo y la gerencia -movilizando información decisoria hacia abajo y de retroalimentación hacia arriba-, hoy son reemplazados o integrados a equipos flexibles, capaces de fijarse metas y objetivos, de promover sugerencias y de resolver problemas con rapidez.

En esta reestructuración, la tendencia de las grandes empresas consiste en implementar una gestión de recursos humanos fuertemente articulada a las estrategias de crecimiento de la organización y claramente integrada en los múltiples aspectos que involucra. Se tiende a actualizar los sistemas de relaciones jerárquicas, a revisar el diseño de los puestos con relación a las necesidades productivas, a redefinir las calificaciones, generar políticas de reconversión, implementar incentivos y estimular la participación (Stankiewicz, 1991).

En este sentido, los cambios tecnológicos y organizativos no pueden realizarse sin la experiencia práctica de los trabajadores y una vez implementados generan la necesidad de construcción de nuevas competencias en los actores del trabajo. La diferencia sustantiva entre viejo y nuevo paradigma productivo, se centra en que la producción masiva se caracterizó por el empleo de trabajadores semicalificados y la flexible, por el contrario, está basada en trabajadores calificados que elaboran una variedad de productos diferenciados, lo que implica la utilización cre- ciente del trabajador como un recurso inteligente (Monteiro Leite, 1996).

Adquiere cada vez más importancia el concepto de "competencia social" como el conjunto de capacidades que se desarrollan a partir de tres campos: la autonomía, la asunción de responsabilidades y la comunicación social; de lo que resulta la importancia de integrar estas actitudes en el seno de las competencias profesionales (Zarifian, 1999).

Así, se redimensionan algunas caracteristicas cualitativas e intangibles involucradas en la productividad de la fuerza de trabajo como las capacidades cognoscitivas, motivos y habilidades que agregan valor a la productividad y que son indisociables de la persona que los posee.

Por oposición a los esquemas tradicionales de calificación, este nuevo enfoque acentúa la importancia del compromiso del trabajador con la razón de ser de su tarea. Se pone énfasis en la relación que existe entre el desempeño de una determinada ocupación con las condiciones tecnológicas y productivas en que se desarrollan las tareas y -en particular-con los objetivos e intereses estratégicos de la empresa. La calificación de los trabajadores se define en torno a situaciones histórico-sociales concretas y en función de las interacciones que se dan en el seno de la vida organizacional (Monteiro Leite, 1996).

Si se materializa esta apropiación social del conocimiento, las organizaciones se constituyen en organizaciones orientadas al aprendizaje (learning organizations), capaces de innovación y de una adaptación creativa a los imperativos de un entorno cambiante.

La articulación entre las competencias de los sujetos y las competencias de 
la organización, posibilitan la construcción de "organizaciones calificantes o formativas", las que generan contextos adecuados de aprendizaje $-y$ razones válidas para aprender- para sus miembros, desde la certeza de que la capacidad de aprendizaje de la organización es el elemento mayor de su eficiencia (Rojas, 1999).

Gestionar el factor humano en un contexto de trabajo flexible implica el desafío de optimizar globalmente el valor que agrega el personal a los procesos productivos. El movimiento que puede propiciarlo es esencialmente de profesionalización de la gestión y se despliega en un escenario socioeconómico en el que escasean los incentivos tradicionalmente ligados al "progreso", por lo que "el aprendizaje y el desarrollo personal y grupal" constituyen una apuesta que potencia las posibilidades de supervivencia en el mercado de trabajo.

Las empresas han comenzado a involucrarse más activamente en la formación, aún cuando los resultados que arrojan las investigaciones realizadas en distintos sectores de la industria latinoamericana y argentina muestran un conjunto variado de trayectorias organizacionales, con distintos alcances de abordaje del tema en el marco de un conjunto de problemáticas compartidas.

En nuestro contexto, sin embargo, las tendencias descriptas aún no se han consolidado. No todas las empresas tienen conciencia del desafío de construir organizaciones inteligentes y cuando lo tienen no siempre pueden emprender un proceso de cambio sistemático y profesionalizado. Factores como su posición en el mercado, el origen de su capital y el sector al que pertenecen, su tamaño, el grado de profesionalización de su gestión, la historia de desarrollo, la microcultura interna -entre otros-; influyen para que desarrollen procesos particulares que -más allá de las tendencias hegemónicas- les permiten sobrevivir y crecer.

Las investigaciones realizadas en distintos sectores empresariales coinciden en que desde hace algunos años las empresas argentinas se vuelcan a desarrollar nuevas estrategias respecto de la concepción de sus negocios, de la organización de la producción y del trabajo, pero muchas son renuentes a cambiar su filosofía y políticas de gestión de recursos humanos. En muchos casos, se mantiene en los dirigentes una "mentalidad no productivista" que paraliza la innovación (Rojas, 1995).

Del mismo modo, es frecuente que los conceptos de flexibilidad sean incorporados en las organizaciones en un sentido restringido, limitando su alcance real o reduciendo su significación social, asimilándola exclusivamente a la idea de "flexibilización de contratos de trabajo". Cuando la flexibilidad es remitida casi exclusivamente a la precarización laboral, su alcance se empobrece. En su sentido más potente, la flexibilidad puede ser entendida como posibilidad de aprendizaje del colectivo social y plantea el desafío de generar dispositivos que promuevan el desarrollo humano en los nuevos escenarios organizacionales.

En lo que respecta específicamente a la capacitación, los desarrollos de investigación centrados en América Latina y el Caribe (Labarca, 1999; Novick, 1999) señalan que los desafíos más apremiantes son la asignación eficiente de recur- 
Reconversión productiva y capacitación del personal en el sector lácteo en Argentina Pujol, Andrea

sos, el diseño de políticas diferenciadas y su implementación efectiva y el diseño de estrategias de formación para sectores deficitarios.

Las investigaciones permiten constatar que la asignación de recursos a la capacitación por parte de las empresas es insuficiente, especialmente si se tienen en cuenta los estándares establecidos por los países desarrollados; a lo que se agrega que la utilización de dichos recursos suele ser ineficiente. Algunas de las razones de esta ineficiencia son:

- dificultades en la detección de necesidades de capacitación;

- oferta inadecuada respecto de las necesidades de la empresa;

- alto costo de la actividad (tanto a nivel del costo directo como del costo emergente de la mediación de instituciones capacitadoras);

- falta de "cultura de capacitación".

En cuanto al diseño de políticas, existe la tendencia a diseñar políticas homogéneas que no tienen en cuenta las diferencias particulares de los distintos procesos productivos y de sus fases de evolución. Esto aparece asociado a tres factores: la dificultad que tienen las empresas para detectar necesidades de formación, antes mencionada, el divorcio entre el área de capacitación y las unidades de producción y la rigidez de los sistemas o planes de formación.

Los estudios sobre reconversión productiva y cambio tecnológico muestran que uno de los problemas para la detección eficaz de necesidades de capacitación parece focalizarse en el carácter adaptativo que adquieren los procesos de innovación. Las empresas, aún las que capacitan, abordan las innovaciones descansando fuertemente en la fuerza adaptativa de la tecnología. Muy pocas empresas de nuestro medio desarrollan actividades de investigación y desarrollo en la región, por lo que los procesos de cambio son implantados como transferencia desde otros contextos. En este marco, suele suceder que el personal deba adaptarse a la innovación sin disponer de una base conceptual. Las competencias requeridas por la innovación, no solo no están disponibles en los sujetos sino que tampoco son fácilmente detectables como necesarias por la gestión de recursos humanos.

Otro problema reside en la modalidad decisoria de las empresas respecto del planteo de un programa de formación. Con frecuencia, las empresas compran la formación según la oferta de mercado, formulando la demanda en los términos establecidos por la oferta (Labarca, 1999); especialmente aquellas que no pueden disponer de su propio sistema de formación. En tal sentido, se reconoce la necesidad de capacitar, pero como se carece de personal idóneo, procedimientos adecuados o herramientas eficaces para detectar necesidades, se suele dejar en manos del oferente (se trate de una consultoría, una institución o una agencia de formación) la detección de necesidades y diseño de propuestas, lo que no necesariamente siempre sive para cubrir las verdaderas necesidades $y$ expectativas de los usuarios directos e indirectos.

En este marco, las salidas a esta situación parecen centrarse en tres cuestiones: el mejoramiento de la articulación entre el sistema educativo y el productivo; el incre- 
mento de inversión en formación -tanto por parte de las empresas como del Estado- y un sustantivo mejoramiento de la ingeniería de la capacitación (Paín, 1996); es decir la utilización sistemática de procedimientos y herramientas eficaces, tanto para relevar necesidades como para diseñar y evaluar los programas de formación. Este diseño debe considerar con igual importancia todos los aspectos: los contenidos, la metodología, los recursos, los resultados a obtener, etc.

Desde este contexto teórico y bajo estos supuestos, se consideró esencial reconstruir la problemática a la luz de las características particulares del sector lácteo y de las empresas que lo componen.

\subsection{Particularidades de las empresas lácteas y su incidencia en la problemática en estudio}

Entre las empresas indagadas, fue posible identificar tres escenarios (ver Tabla 1): un subgrupo de tres empresas ( $A, E$ y G) que -aún con características distintivas- lideran el sector, por su importancia en el mercado, la complejidad de su estructura y recursos, su dinámica de innovación y la presencia de un management más profesional. Una caracteristica importante de este grupo es que no han sufrido procesos de fusión con otras empresas o de incorporación de capitales desestabilizadoras, sino que han vivido procesos de innovación técnica y organizacional, derivados de su propia evolución organizativa a modo de "saltos cualitativos" en su historia. Esta particularidad es señalada recurrentemente por dirigentes y trabajadores, quienes perciben que la empresa ha cambiado sustantivamen- te pero que conserva algunos rasgos propios de su conformación histórica -para bien o para mal-.

El segundo subgrupo, lo componen tres empresas medianas $(B, C, D)$, que han atravesado cambios de firma con una serie de efectos de desestabilización de su dinámica interna. En esas empresas se observó menor claridad y consistencia en cuanto a estrategias de competitividad y una filosofía de gestión de recursos humanos escasamente compartida. Los procesos de cambio de firma a los que han estado sujetas son muy recientes, por lo que ha habido cambios de estrategia frecuentes y una alta rotación a nivel gerencial que hace que dirigentes y trabajadores perciban a la empresa "sin un rumbo claro" o "en transición".

Por último, una empresa cooperativa pequeña $(F)$ que aún no ha realizado mejoras sustantivas a su tradicional modo de operar y que se encuentra en proceso de alianza estratégica con una de las del primer subgrupo.

En el desarrollo del estudio pudo constatarse el dinamismo del sector: dos de las empresas (empresas $B$ y $E$ ) realizaron alianzas estratégicas con otras empresas del sector, la empresa G cerró una de sus plantas, la empresa $E$ abrió dos nuevas plantas, la empresa $\mathrm{C}$ compró las acciones de otra más pequeña, etc. Una de las empresas (empresa D) de origen local, por ejemplo, fue explorada en la etapa de inserción del equipo de investigación, en el momento en que se hallaba en negociaciones para vender la mayoría de sus acciones a un grupo económico de capital mixto, negociación que se concretó seis meses después. El seguimiento de este proceso de transferencia de capi- 
Reconversión productiva y capacitación del personal en el sector lácteo en Argentina Pujol, Andrea

\section{Tabla 1}

Información general sobre las empresas encuestadas

\begin{tabular}{|c|c|c|c|c|c|c|}
\hline Empresa & $\begin{array}{c}\text { Cantidad } \\
\text { de Fabricas }\end{array}$ & Antigũedad & $\begin{array}{c}\text { Origen } \\
\text { actual del } \\
\text { capital }\end{array}$ & $\begin{array}{l}\text { Forma } \\
\text { societal } \\
\text { actual }\end{array}$ & $\begin{array}{c}\text { Producción } \\
\text { aproximada } \\
\text { en litros } \\
\text { diarios } \\
\end{array}$ & $\begin{array}{c}\text { Cantidad } \\
\text { de personal }\end{array}$ \\
\hline$A$ & 10 & 70 años & Nacional & Familiar & $1.000 .000 \mathrm{l} / \mathrm{d}$ & 1000 \\
\hline B & 1 & 60 años & Nacional & Soc. Anon. & $600.000 \mathrm{l} / \mathrm{d}$ & 475 \\
\hline $\mathrm{C}$ & 1 & 50 años & Extranjero & Soc. Anon. & $650.000 \mathrm{l} / \mathrm{d}$ & 400 \\
\hline D & 3 & 50 años & Mixto & Soc. Anon. & $200.000 \mathrm{l} / \mathrm{d}$ & 230 \\
\hline$E$ & 21 & 60 años & Nacional & Cooperativa & $6.000 .000 \mathrm{l} / \mathrm{d}$ & 5000 \\
\hline $\mathrm{F}$ & 1 & 70 años & Nacional & Cooperativa & $300.000 \mathrm{l} / \mathrm{d}$ & 230 \\
\hline G & 7 & 86 años & Extranjero & Soc. Anon. & $1.800 .000 \mathrm{l} / \mathrm{d}$ & 3000 \\
\hline
\end{tabular}

Fuente: empresas encuestadas. Elaboración propia.

tales permitió realizar inferencias significativas respecto de la problemática en estudio.

En el marco de estos cambios, el origen de capital y la forma societal constituyen dos factores de importancia en lo relativo a la capacitación, con una influencia notable en el grado de profesionalización de la gestión de recursos humanos y en el desarrollo de las acciones de formación.

La gestión de recursos humanos en las empresas de origen nacional es de reciente implantación. Tradicionalmente, estas empresas contaban solo con una oficina de personal en la que se realizaban actividades de administración de sueldos y algunas acciones en lo relativo a seguridad, higiene industrial y relaciones laborales. Entre 1994 y 1996 se inicia un proceso de profesionalización de la gestión -que en muchas empresas es aún incipiente-, que implicó la puesta en marcha de algunas acciones de capacitación
- ligadas en general a los procesos de certificación de calidad- y en menor medida una revisión de los mecanismos de reclutamiento y selección del personal.

Sin embargo, aún dentro de las empresas de origen nacional, cabe destacar algunas diferencias en cuanto a gestión de recursos humanos que derivan de su forma societal: tanto en el caso de las cooperativas como en el de la empresa familiar -aún cuando no existía una gestión profesionalizada de recursos humanos-, se atendió siempre lo relativo a beneficios sociales y acciones de educación no formal, como becas para hijos de empleados y actividades deportivas y recreativas.

Cabe destacar también que dos de las sociedades anónimas -una con capitales nacionales y otra recientemente adquirida por un grupo internacional- se definieron -en la voz de sus directivoscomo "de gestión familiar", entendiéndose por ello "es una gestión casera, pero 
cuidamos mucho a la gente" (EA) ${ }^{3}$ y "hacemos todo intuitivamente, por olfato, pero atendemos las necesidades de la gente, porque vivimos en la misma comunidad, porque gran parte de la gente que vive aquí trabaja en la empresa"(EC).

En la mayoria de las empresas se observó una fuerte vinculación con la comunidad. La ubicación de las plantas industriales en ciudades pequeñas o medianas fortalece las relaciones con el entorno, a lo que se suma en otros casos la forma societal, cuando se trata de cooperativas de tamberos, (productores de leche cruda) quienes generalmente desarrollan su actividad en la zona en la que se encuentran las fábricas. Sólo constituyen excepciones las plantas radicadas en zona urbana (EF) y otra cuya inserción en la comunidad es problemática desde hace muchos años (EG).

Del mismo modo, resulta interesante destacar la importancia que adquieren para las empresas las actividades de "asesoramiento al productor", en la medida en que la calidad de la materia prima fue considerada en los últimos años el factor clave de competitividad del sector. El asesoramiento y capacitación a los productores ocupa un importante lugar en la estructura de las empresas seleccionadas -aún cuando no se trate de cooperativas de tamberos-, en contraste con una estructura muy pequeña, destinada a la "adminis- tración de personal" y más pequeña aún en lo relativo a capacitación de los recursos humanos.

En este sentido, en los documentos consultados sobre la dinámica del sector lácteo se destaca que el tema de la calidad de origen de la materia prima ha cobrado significativa importancia en los últimos años. Según los empresarios "no existe proceso de industrialización que pueda mejorar la calidad de origen de la leche"(EE), y si bien la innovación tecnológica y la implementación de normas de calidad industrial inciden sobre la competitividad, el factor clave sigue siendo la materia prima. En este marco, la importancia asignada a la competencia del personal como "factor que agrega valor al producto"(EA) es bastante relativa.

"La evolución de la empresa láctea comenzó resolviendo problemas en relación a la calidad de la materia prima, por eso tenian un gran departamento de asistencia al productor, al tambero. Hoy se observa en las estructuras empresariales la desarticulación de estos departamentos (...) por ejemplo en el departamento de asistencia primaria antes había siete personas, hoy hay una sola. Antes había en todas las empresas una estructura amplia y fuerte en producción primaria y muy pobre en recursos humanos, ahora estamos notando un cambio." (Entrevista al director del departa-

3 Para indicar las fuentes de las expresiones codificamos a las empresas como EA, EB, EC... etc., según la Tabla 1. 
mento de capacitación y extensión de la ESIL $\left.^{4}\right)$.

A su vez, el gerente de una de las empresas cooperativas sostiene:

"Nuestra empresa tiene capacitación a distintos niveles, una es la capacitación al productor, que es la base de sustento de la cooperativa, en esa línea hay una capacitación que ejerce la fundación, por un lado y la Federación de Centros Juveniles, por otro. Esto es una capacitación, fundamentalmente a los hijos de los productores para ir preparando de alguna manera a los futuros dirigentes cooperativistas zonales. Este es el enfoque hacia al nivel de productores, es una capacitación selectiva, de hecho hay toda una serie de programas, hay encuentros formales directos en cada una de las zonas, la presencia de especialistas de determinadas cuestiones clave en la labor diaria que tiene el productor, esto es introducir al joven dentro de la tarea que los padres - abuelos vinieron realizando con un gran sentido del esquema cooperativista, esa es la base de partida de lo que hace en función de nuestra gente que termina siendo en la práctica los propios dueños de esta empresa, de ahi que ustedes van a encontrar una filosofía distinta entre lo que es una empresa familiar, una empresa de otro tipo, digamos privada (...) Des- pués estamos con la capacitación orientada a los dirigentes, o sea a los integrantes del consejo directivo de esta empresa que está conformado por aquellos productores que en cada una de las zonas comarcales son elegidos por sus propios colegas para que los representen en este consejo o directorio, también ahí se hace una capacitación a medida, de manera de orientarlos en cuanto a que aquella actividad (la de productores) no es tan lo mismo como ésta. (gerente de recursos humanos $\mathrm{EE}$ ).

Al igual que la estructura legal, los productos que se elaboran y las características particulares de los procesos productivos, inciden también sobre la dinámica interna de gestión de los conocimientos.

En este aspecto, los productos que fabrican las empresas del sector (ver Tabla 2) son comunes a la mayoria, a excepción de una de ellas que completa su producción con una gran cantidad de productos que podrían incluirse en el sector alimentario (EG).

El proceso de producción se rige por ciclos naturales y parte de su consumo depende de las variaciones climáticas, lo que influye sobre la programación de la producción, la mano de obra requerida y la organización del trabajo. Tam-

4 La ESIL (Escuela Superior Integral de Lechería de Villa Maria. Córdoba. Argentina) es la institución capacitadora por excelencia de la mano de obra del sector a través de cursos que desarrollan en las empresas como actividad extensionista. A su vez, la escuela dispone de una oferta educativa formal de nivel superior compuesta por una carrera de nivel terciario que otorga el título de Técnico en Lechería a los dos años y el de Técnico Superior en Lechería y Tecnología de Alimentos (carnes, frutas, hortalizas, oleaginosas, cereales, panificación) a los cuatro años. A su vez, en articulación con la Universidad Nacional de Villa María se ha implementado la carrera de Ingeniería de Alimentos, hace dos años. 
bién cobran mucha importancia el desarrollo tecnológico y su impacto en las prácticas de capacitación, como así también la existencia de formas de organización del trabajo y/o tecnologias de gestión que favorecen la participación y el aprendizaje organizacional, aún cuando se dan en muy pocos casos.

A nivel de insumos y productos, se observa poca variedad de insumos productivos y los productos finales son simples y muy diferenciados, lo que implica la existencia de varios procesos muy diferentes entre si.

En el ambiente físico de las plantas se observa poco personal, disperso en instalaciones de medianas a grandes, 10 que limita la interacción grupal y condiciona las oportunidades y posibilidades de capacitación entre pares.

Como se dijo antes, las características del proceso de producción y el diseño del ambiente físico en que se desarrollan las tareas, se consideran relevantes en la problemática en estudio. Las industrias del sector lácteo se caracterizan por la combinación de procesos artesanales -como la fabricación de algunos quesos o dulces-, con procesos físicos continuos como el de la producción de leche fluida y con procesos de envasado $-u$ hojalatería, en un caso- y de embalaje en serie.

En relación a este punto, es importante considerar que la variedad de productos elaborados impacta en la problemática de la capacitación de manera notable, ya que existe una relación bastante estrecha entre la diversidad de productos elaborados, la mano de obra requerida, sus competencias, las posibilidades de aprendizaje y la capacitación recibida.
En las empresas observadas fue posible identificar que en los procesos cuasi artesanales -si bien se trata de la minoría de los puestos de trabajo-, subsisten los "oficios" (ej: el dulcero) y la experiencia de los trabajadores, su conocimiento acumulado sobre el producto y el proceso, es fuertemente valorado. En general, el personal afectado a estos procesos es el de más edad, tiene mucha antigüedad en la empresa y bajo nivel de educación formal. Salvo en una de las empresas relevadas, estos trabajadores no están sometidos a rotación interna, ni se les incluye en los programas de desarrollo de polifuncionalidad y polivalencia.

En cuanto a la capacitación, los trabajadores más antiguos operan como formadores de los mas jóvenes pero no son asistidos con capacitación, salvo cuando se realiza alguna innovación tecnológica puntual. En algunos casos, incluso, esta innovación puede significar la desvinculación del trabajador, en el caso de que no se lo considere "reconvertible" mediante la capacitación; en algunos casos por la edad, en otros por falta de confianza en su potencial, en otros por el escaso tiempo que les resta para jubilarse.

Tradicionalmente estos puestos significaban un lugar de llegada en la trayectoria de un trabajador dentro de la empresa y el acceso a ellos implicaba la adquisición de cierto status y la consolidación de la identidad profesional, el acceso a una calificación, a un "oficio". Actualmente tienden a desaparecer, y en el discurso de la empresa -no asi en el colectivo de trabajo- son desinvestidos de la importancia que antes se les asignaba y se desmitifican continuamente sus aportes, 


\section{Tabla 2}

\section{Principales productos elaborados por las empresas}

$\begin{array}{cl}\text { Empresa } & \text { Producción Principal } \\ \text { A } & \begin{array}{l}\text { Manteca, dulce de leche, leche en polvo entera y descremada, quesos } \\ \text { pasta blanda, semidura y dura, queso rallado, leche larga vida, leche } \\ \text { concentrada. }\end{array} \\ \text { B } & \begin{array}{l}\text { Leche en polvo entera y descremada, quesos pasta dura, semidura y } \\ \text { blanda, quesos untables, queso rallado, crema, manteca. }\end{array} \\ \text { C } & \begin{array}{l}\text { Quesos, manteca, crema, dulce de leche. } \\ \text { D }\end{array} \\ \text { E } & \begin{array}{l}\text { Manteche fluida, productos frescos, quesos, dulce de leche, crema. } \\ \text { leche, yogures, suero de queso en polvo, proteinas de leche. }\end{array} \\ \text { F } & \text { Leche fluida y productos frescos. } \\ \text { G } & \begin{array}{l}\text { Leche fluida y en polvo, frescos, quesos, manteca, helados, dulce de leche, } \\ \text { postres, flanes y otros productos alimenticios como cereales, chocolates y } \\ \text { café. }\end{array}\end{array}$

Fuente: empresas encuestadas, Elaboración propia.

bajo el argumento de que la innovación tecnológica y los procedimientos de calidad hacen que nadie sea imprescindible.

"Los recursos tecnológicos se encuentran a la vuelta de la esquina y eso hace que nadie sea imprescindible... antes por ejemplo el dulcero o el que hacía la ricotta eran gente especial, habia que cuidarla como al pan... toda la fábrica estaba convencida de que nadie, absolutamente nadie hacia el dulce como el viejo Juárez. Hoy está todo procedimentado... lo hace bien Juárez, Gómez, o Pérez... cualquier puesto es igual que otro $y$ en definitiva cuando Juárez se fue pusimos un chico joven y se las rebuscó. Con otros dos hombres, mantequeros, pasó más o menos lo mismo: cuando trajimos la línea nueva hubo que decidir si se los capacitaba o si se los desvinculaba. En un caso no tenia sentido porque le faltaban dieciocho meses para jubilarse, así que le mandamos el sueldo a la casa hasta que se jubi- ló. En el otro caso, bueno, era más diffcil... era un hombre muy limitado y tenía casi cincuenta y tres años... no tenía mucho sentido enseñarle a usar las máquinas. Creo que al final se lo pasó a un sector de quesería de la planta vieja porque faltaba gente. En el caso de fabricación no tenemos esos problemas: hoy ven todo el proceso en una pantalla y mañana va a ser una caja negra. Por eso tienen que ser buenos en el manejo de información". (jefe de producción ED).

En los procesos físicos continuos (producción de leche) y en laboratorio, en cambio, se agrupa la mayoria del personal: jóvenes con escasa antigüedad, nivel de escolarización terciario -en general especializados en lechería-, e incluso algunos estudiantes universitarios $y / 0 \mathrm{pa}-$ santes. En este caso se valora fuertemente la educación formal y las competencias requeridas pasan más por los conocimientos y las actitudes que por las 
habilidades (se valora especialmente la capacidad de producir, interpretar y procesar información). Estos trabajadores forman parte de los programas de gestión de competencias, de polivalencia y polifuncionalidad y son los más asistidos mediante la capacitación, especialmente en 10 relativo a temas de calidad (filosofía, gestión y herramientas), dominio de nuevas tecnologías, técnicas de control estadístico y capacítación actitudinal (trabajo en equipo, comunicaciones, etc.).

En el sector de envasado predomina personal joven, con nivel medio, sin especialización en lechería (bachiller, comercial, técnico). Estos procesos se encuentran casi totalmente automatizados -salvo por ejemplo el envasado de yoghurt con frutas o cereales, que es más complejo-, y requieren especialmente de competencias instrumentales, habilidades centradas en la velocidad, la precisión de movimientos, etc.

En este sector también es frecuente la rotación por distintos puestos de trabajo pero además, el personal es menos estable y se dan con frecuencia los contratos a término o de aprendizaje. Se observó también en tres de las siete empresas que gran parte del personal del sector de envase se encuentra estudiando alguna carrera terciaria o universitaria y es frecuente que consideren su trabajo como "una tarea fácil, bastante mecánica, que no me obliga a estar con toda la cabeza acá... por ahi tuve otros trabajos que no me permitian estudiar, que me exigían... este es un trabajo piola ${ }^{5}$, aunque por ahi hay momentos en los que se complica" (EG).

En el caso del sector de envasado, la capacitación suele apuntar especialmente al dominio de máquinas y sigue su ciclo: se incrementa e intensifica ante las innovaciones y se paraliza en periodos de escasa innovación. En este sector el entrenamiento en el puesto -desarrollo de habilidades- es muy intenso al momento del ingreso del trabajador y es clave para determinar su permanencia en la empresa.

"Acá se necesita poco conocimiento, sólo que vean bien la fecha que se coloca (de elaboración y vencimiento)... el conocimiento no es tan prioritario porque se lo damos nosotros... lo que veo es el movimiento de la persona... cómo se comporta, la voluntad que tiene, el emprendimiento... el que lo hace por rutina va a seguir poniendo quesos en la cinta... si da para más va a pasar a elaboración... Siempre veo el emprendimiento que tiene, pero tampoco que se pase de vuel$\operatorname{tas}^{6}$, que vaya de acá para allá, eso es peligroso, también es peligroso para él...". Señala que el que está en la máquina envasadora tiene un poco más de conocimientos que los que sólo ponen quesos en la cinta o embalan al final de la línea): "el que está en la máquina si tiene que te-

6 Se refiere a que haga cosas que no le corresponden por su función y corra riesgos por realizar operaciones inseguras con las máquinas. 
ner conocimientos, para poder manejarla..." (supervisor envase de quesos EB).

\subsection{La capacitación en el marco de los imperativos de las empresas y el sector}

En función de la información relevada, los desafíos que enfrentan las empresas (ver Tabla 3) pueden ser considerados factores impulsores de acciones de capacitación, aún cuando no se constituyen en bases para el desarrollo de políticas a largo plazo.

En este sentido, las empresas mencionan tres desafíos de modo recurrente: centificar calidad, lograr una mejor inserción y distribución de los productos en el mercado y reducir costos. En segundo término, se consideran: incrementar la productividad, incrementar las ventas $y$ exportar.

\subsubsection{El desafío de la calidad}

En los últimos años, la certificación de calidad forma parte de las estrategias de las empresas del sector para insertarse o permanecer en el Mercosury en el mercado internacional. El proceso de implementación de las normas es un proceso complejo que requiere de nuevos aprendizajes para la organización y también de la inversión de tiempo y recursos económicos en capacitación del personal.
La certificación de calidad ${ }^{7}$ le permite a la empresa contar con la documentación de todas las actividades relativas a la calidad, por escrito: manuales de calidad, procedimientos generales y especificos, registros, etc. y con ello estandarizar las tareas y actividades. Al documentar los procedimientos, se debe incluir el cómo debe realizarse cada una de las actividades y quién es el encargado de realizarlas, pero la propia empresa es la que debe decidir el mejor sistema para cumplir con los requisitos de la norma.

Cuando el proceso de implementación de normas de calidad resulta eficaz se transforma en una oportunidad para desarrollar un programa sistemático de capacitación a corto, mediano y largo plazo, e incluso hace que la capacitación se constituya en un valor de la cultura de la empresa.

En este sentido, la serie de las Normas ISO 9000 en sus diferentes capítulos (tanto en los apartados sobre "generalidades de la capacitación" como particularmente en el capítulo dieciocho referido a los diferentes aspectos de la gestión del personal) expresan la importancia de identificar necesidades de capacitación y de capacitar a todo el personal, manteniendo registros adecuados de las actividades. También se hace referencia a la necesidad de optimizar el reclutamiento y la selección del personal y la asignación de tareas, considerando los niveles eduorganizacional, responsabilidades, procedimientos, procesos y recursos, certificables por una tercera parte, que debe reunir una empresa para la implantación de un Sistema de Asegura- 


\section{Tabla 3}

\section{Principales desafios que enfrentan las empresas}

\begin{tabular}{|c|c|}
\hline Empresas & Principales Desafíos \\
\hline A & $\begin{array}{l}\text { Lograr una mejor inserción y distribución de los productos en el mercado } \\
\text { Aumentar las exportaciones } \\
\text { Certificar calidad }\end{array}$ \\
\hline $\mathrm{B}$ & $\begin{array}{l}\text { Lograr una mejor inserción y distribución de los productos en el mercado } \\
\text { Aumentar la productividad } \\
\text { Reducir costos }\end{array}$ \\
\hline $\mathrm{C}$ & $\begin{array}{l}\text { Incrementar las ventas } \\
\text { Aumentar las exportaciones } \\
\text { Certificar calidad }\end{array}$ \\
\hline$D$ & $\begin{array}{l}\text { Reducir costos } \\
\text { Desarrollar nuevos productos } \\
\text { Lograr una mejor inserción y distribución de los productos en el mercado }\end{array}$ \\
\hline$E$ & $\begin{array}{l}\text { Lograr una mejor inserción y distribución de los productos en el mercado } \\
\text { Aumentar las ventas } \\
\text { Certificar calidad }\end{array}$ \\
\hline$F$ & $\begin{array}{l}\text { Aumentar la productividad } \\
\text { Reducir costos } \\
\text { Certificar calidad }\end{array}$ \\
\hline $\mathbf{G}$ & $\begin{array}{l}\text { Reducir costos } \\
\text { Aumentar la productividad } \\
\text { Incrementar las ventas }\end{array}$ \\
\hline
\end{tabular}

Fuente: Elaboración propia.

cativos y competencias como criterios sustantivos.

En la mayoria de las empresas estas recomendaciones han operado como motor para iniciar acciones de capacitación. Sin embargo, las normas no especifican la capacitación requerida ni se dan los lineamientos para identificar las necesidades de capacitación, por lo tanto es posible que se registre la capacitación en base a criterios no necesarios, o que se omitan las necesidades básicas de capacitación. En este sentido, las normas recomiendan que la dirección determine los niveles de competencia, experiencia y capacitación exigidos por las tareas.

En lo relativo a los supervisores, se acentúa además la importancia de que sean capacitados en los procedimientos y habilidades requeridas para efectuar sus tareas, considerando los instrumentos, herramientas y maquina-

miento de la Calidad destinado a proveer confianza a sus clientes en el cumplimiento de los requisitos de calidad acordados. Los sistemas de Aseguramiento de la Calidad permiten detectar cualquier defecto de producción antes de que el producto llegue al mercado o corregir las fallas observadas posteriormente para evitar que se repitan, contribuyendo además a reducir costos improductivos para lograr un precio final más bajo. 
rias que se utilizan, la lectura de documentación, etc.

A su vez, en diferentes apartados señalan cuestiones relativas a la calificación y la motivación, acentuando la necesidad de asentar los niveles de calificación y evaluarlos periódicamente, de revalorizar el dominio de la tarea como factor motivacional, de capacitar actitudinalmente facilitando el desarrollo de "conciencia de calidad" y de proporcionar reconocimiento al desempeño.

En el sistema ISO 9004, los puntos anteriores se establecen como recomendaciones y es muy conveniente que se apliquen, ya que sirven para facilitar la implementación de las normas certificables; pero no son de ninguna forma requisitos obligatorios por ejemplo para las normas 9001,9002 y 9003 . Sin embargo, la mayoría de los dirigentes son conscientes de que los trabajadores juegan un papel importante en el éxito o fracaso de un sistema como ISO 9000, y que son en cierto modo garantes de la confiabilidad del sistema de calidad.

En este sentido, se hace visible que nadie mejor que el trabajador para encontrar los puntos críticos y las oportunidades de mejora en el proceso, porque él es quien tiene un mayor conocimiento de las actividades que realiza y de la maquinaria que utiliza día a día y en ocasiones gran parte de la calidad se encuentra en manos de los operadores, quienes deben reducir los desperdicios, eliminar los tiempos muertos y hacer buen uso de las máquinas y de los materiales que utilizan.

Si bien es cierto que la certificación en ISO incluye nuevos aspectos en lo relativo a la gestión de las competencias del personal, también es posible afirmar que las competencias que exige ISO -y por ende las que se buscará generar con la capacitación-, están sujetas a lo que se establezca en la documentación, por lo que todo depende en gran parte de la efectividad de la modalidad con la que la empresa implemente el sistema. Además, la exigencia de ISO no implica necesariamente que se reconozca el logro individual de los trabajadores en torno a la mejora continua, a la polifuncionalidad y a la polivalencia.

En el caso de las empresas relevadas, seis de las siete empresas tienen procesos de certificación concluidos y una de ellas recién iniciado. Sin embargo, al interior de cada empresa la situación no es homogénea: los procesos de implementación se inician generalmente por las plantas cuya producción está destinada a exportaciones y la mayoria de las empresas sólo tienen algunas de sus plantas con sistema ISO.

En este marco, la cantidad y frecuencia de actividades de capacitación se incrementa en las plantas sujetas a ISO y con ello, las oportunidades de participar de actividades de capacitación por parte de los colectivos de trabajadores que se desempeñan en dichas plantas. Como contraparte, en las plantas sin ISO se observan colectivos de trabajadores que rara vez reciben alguna capacitación o que no la han recibido nunca.

Consultado por las razones de los cursos masivos recientes, un operario, facilitador de calidad, responde:

"El primero (curso) fue para que todo el mundo supiera de que se trataba de ISO, era en el horario de trabajo, como era la primera que se hacía, se buscaron a 6 personas y cada uno manejaba un 
grupo de 20, 30 personas, se hicieron muchas reuniones con grupos chicos, era intersectorial, no era en un sector, sino de producción, lecheria, laboratorio, de mantenimiento, cosa que se hiciera más dinámico, por ejemplo hay gente de producción que tenía dudas, que la gente de mantenimiento ni lo hubiese pensado, entonces se sacaron todas las dudas. El de lecheria fue opcional, ese se hacía fuera del horario de trabajo, si querías hacerlo, venías fuera del horario y lo hacías, lo pagaba la empresa, vino muchísima gente. Ahora se está proyectando todo 10 que es $\mathrm{HACCP}^{8}$, lo que $\mathrm{BPM}^{9}$ y Cinco " $s$ "10 entonces se está armando una capacitación para toda la planta de eso, esta es como la primera, va a llegar a todos, porque son pre-requisitos que hay que cumplir entonces todos tienen que estar enterados, cuáles son los puntos críticos, qué se hace en cada punto críti$\mathrm{co}$, cómo tienen que trabajar ellos con lo de buenas prácticas, como se ordena, limpia, que es Cinco "s", todo lo que es barrera sanitaria, todo lo tienen que conocer, porque en el momento que vengan a auditar, le van a decir "qué es eso"?, y el operario tiene que saber, no con lujo de detalles como diría el instructivo, pero tie- nen que saber lo que está haciendo, en base al instructivo" (EE).

Otra relación fuerte entre capacitación y certificación de calidad se vincula a la frecuencia e intensidad de las acciones de capacitación: en muchos casos pudo verificarse que las auditorías externas de calidad constituyen el momento clave en que se hacen efectivas acciones de capacitación. En este sentido, la capacitación sigue el ritmo de la importancia estratégica que tiene para la empresa el logro y mantenimiento de la certificación: se realizan actividades frecuentes e intensivas de capacitación en la etapa previa a la certificación y -posteriormente- en las instancias previas a cada auditoría, mientras hay importantes periodos de tiempo en que no se realiza ningún tipo de actividades.

Esta situación es percibida con claridad en los distintos niveles y de algún modo contribuye a que exista cierto descreimiento acerca de las nomas ISO.

"En realidad la capacitación está supeditada al interés de lograr la certificación ISO 9000, porque uno de los requisitos para lograr esta certificación es el realizar cursos de capacitación. Por eso se hicieron ya en leche en polvo y ahora en

El sistema HACCP (Hazardous Analysis and Critical Control Point) es una herramienta de última generación que garantiza la calidad de los productos que consume el cliente. El Hazard analiza los puntos críticos de control y permite corregir cualquier desperfecto antes de que el producto llegue al punto de venta. Instrumentos como éste son imprescindibles en la producción de alimentos.

9 Buenas Prácticas de Manufactura.

10 El sistema Cinco " $\mathrm{S}$ " es parte de la filosofía japonesa para el mejoramiento continuo y consiste en un procedimiento sencillo estructurado por las claves: Ser organizado, Ser Ordenado, Ser limpio, Ser Uniforme, Ser Disciplinado. (En japonés: Seire, Sciro, Seiketsu, Shitsuke) 
$U \mathrm{HT}^{11}$. Yo sostengo que esto de ISO 9000 es un barniz que le pusieron, si rascás un poco, más abajo no hay nada... la gente hace las cosas, pero no sabe por qué... vas a ver que tienen todo escrito en unas carpetas asi (me muestra el tamaño, exagerándolo), y que las tienen en el escritorio, pero en el fondo no entienden lo que están haciendo... Los encargados somos los que recibimos el doble discurso... desde arriba nos dicen tienen que capacitar', pero a la vez no nos hacen el tiempo... Yo te puedo asegurar que si los capacitás, esta gente aprende... "(encargado de mantequería EA).

"En realidad en la empresa no hay mucho escrito, formalizado, sobre el tema de planificación y objetivos en recursos humanos... eso no quiere decir que no exista, lo que pasa es que está implícito... acá la comunicación es mucho más oral que escrita... Yo hice un intento por empezar a tener todo por escrito, pero no funcionó: nadie lo leía... al final gastaba un montón de tiempo en eso y después nadie lo leía. En realidad hace un año que empecé a dejar por escrito la planificación de recursos humanos y una de las cosas que están por escrito es la planificación de capacitación de 1996 y 1997. Incluirnos en ISO 9000 nos obligó en alguna medida a tener por escrito la planificación de la capacitación, pero por ahi la capacitación se hace toda junta antes de la auditoría... y eso no es bueno ni es calidad". (responsable de recursos humanos $\mathrm{EB}$ ).

En este marco, se pudo observar que la presencia de programas de calidad en las distintas fábricas no siempre implica una modificación sustantiva de las operaciones y de las formas históricas de actuar de los trabajadores. En muchos casos observamos que se trata de una "operación discursiva" (Catalano y Hernández, 1994), que pretende "cambiar el tipo de mirada sobre la empresa y hacer visibles problemas que antes permanecian ocultos", aún cuando no siempre se concreta en las acciones y sólo queda en el nivel de un discurso de moda.

En el tema de la calidad, nuestra mirada en las empresas del sector lácteo coincide con algunas apreciaciones que realizan al respecto otros investigadores: "En torno a la calidad hay sin duda más discurso que acción y más teoría que práctica... sólo el $1 \%$ de los programas de calidad aciertan, mientras que los demás naufragan dejando un rastro de desánimo y frustración. De todos modos, la calidad parece haberse transformado, para las empresas de los años 90 , en lo que la microelectrónica terminó siendo a comienzos de los 80: un catalizador de estrategias productivas y un recurso de marketing. Hace diez años, los adeptos a las máquinas de comando numérico afirmaban: llega un momento en que tener el comando numérico es más importante

11 Se entiende por Leche UAT o UHT (Ultra Alta Temperatura) a la leche homogeneizada, que ha sido sometida durante 2 a 4 segundos a una temperatura entre $130^{\circ} \mathrm{C}$ y $150^{\circ} \mathrm{C}$, mediante un proceso térmico de flujo continuo, inmediatamente enfriada a menos de $32^{\circ} \mathrm{C}$ y envasada bajo condiciones asépticas en envases estériles y herméticamente cerrados. 
que usarlo. Es igual a lo que se encuentra en muchas empresas: lo que cuenta es tener un programa de calidad; si funciona, es otra cuestión" (Leite, 1996).

En este aspecto, resulta significativo indagar en el carácter instrumental que adquieren las herramientas de gestión en los paises emergentes y el papel paradigmático que cumplen en este sentido las ISO.

En la medida en que en América Latina no se producen herramientas de gestión y que la incorporación de las mismas se produce por los mecanismos más diversos, las evaluaciones de las herramientas incorporadas son casi nulas y existen débiles mecanismos de jerarquización y ponderación de las mismas (Ruffier, 1998). Además, al existir redes vinculadas a ciertas familias de herramientas sin que se establezca coordinación con otras, coexisten y se utilizan herramientas de gestión de las más distintas características y lógicas simultáneamente en un mismo espacio físico y social. Aun si ellas tienen principios distintos e incluso contradictorios.

En este sentido, también Ruffier (1998) destaca cierta ausencia de univocidad de criterios en la aplicación y falta de análisis adecuado de cuáles son los posibles mecanismos sociales que estas herramientas ponen en funcionamiento. Asi, la eficiencia de las herramientas resulta mucho más limitada de lo que requieren las empresas y aún más que la que prometen sus difusores. Otra de las consecuencias de esta realidad es que las empresas muchas veces intenten aplicarlas parcialmente, tensionando su lógica interna o las abandonen total o parcialmente para incorporar otras sin una correcta evaluación de por qué no les fueron útiles o por qué no se cumplieron las expectativas puestas en ellas. Esta dinámica en la que entran ciertas empresas debilita la credibilidad general en las herramientas de gestión y a su vez es una fuente de despiffarro y de falta de eficiencia.

\subsubsection{Los desafíos comerciales}

La gestión de marketing y la gestión de exportaciones constituyen otro desafío importante de las empresas, pero la vinculación de este desafío con la problemática de la capacitación no es lineal.

Si bien sólo en un caso fue destacada el área comercial como aquella en la que se vuelven obsoletas las competencias del personal, cuando las empresas fueron consultadas respecto de las áreas que son más atendidas con actividades de capacitación, tres de ellas dieron importancia al área comercial, junto con la de producción ( $E B, E C$ y EE).

Estas tres empresas justifican estas acciones en la necesidad de mantenerse actualizadas en el tema de marketing y logística comercial, en función de la competencia creciente y de la ampliación de mercados que supone el Mercosur. La empresa $B$, además, señaló que una de las razones que habia conducido a la empresa a buscar aliarse con otra empresa era su "incapacidad en lo comercial" (EB), por lo que intentan permanentemente mantener "activa a la gente" con capacitación.

Las otras empresas consideran que es en el área de producción y en el área de administración en donde las competencias se vuelven obsoletas más rápida- 
mente. En el primer caso, por la innovación tecnológica y por la implementación de calidad; en el segundo, por la innovación en tecnologías de información. También señalan las áreas de producción y administración, como las áreas más atendidas en lo relativo a capacitación.

En relación al desafío de reducir costos, no fue posible establecer una vinculación clara y directa con la capacitación. Esto obedece sin embargo a causas que resulta importante puntualizar. En principio cabe preguntarse qué significado atribuyen las empresas a la expresión "reducir costos", y luego, qué conceptualizaciones sobre este punto pueden servir de orientación para dimensionar dichas significaciones en el marco de este estudio.

En principio, la mayoría de las empresas del sector asocian la reducción de costos a la reducción del costo laboral, costos de materia prima y de operaciones de producción. En cuanto al costo laboral, cuatro de las siete empresas expresan haber logrado reducirlo a través de: incorporación de tecnologías, mejora de la eficiencia y nuevas modalidades de contratación (EA); ajustes y fusiones de estructuras, concentración de procesos e instalaciones de alta tecnificación (EE); ordenamiento de la jornada laboral (EC); incorporación de tecnología y de pasantes (EB).

El tema de la reducción de costos es destacado también en la documentación relativa a la dinámica económica del sector $^{12}$ como obstáculo del desarrollo, tanto para los productores como para las indus- trias: carencia de infraestructura vial, alto costo de la energía potenciado por la distribución a través de cooperativas pequeñas, ausencia de una ley de flexibilización laboral para el sector que permita una reducción de los aportes patronales.

Sin embargo, cabe destacar que algunas empresas consideran también a la capacitación como un costo "en la medida en que si no retorna la inversión, o la gente no logra transferir lo que aprende al puesto... bueno... la capacitación termina siendo un costo" (EF). El retorno de la inversión en capacitación se asocia al nivel de rotación de personal -capacitar a la gente y que luego se vaya de la empresa-, a la edad de los trabajadores -capacitar a trabajadores que por su edad no son "reconvertibles", a la constante innovación tecnológica que vuelve obsoletas muy rápidamente las competencias, o -precisamente- a las dificultades que se visualizan en la transferencia de aprendizajes al puesto de trabajo.

Un estudio sobre cambios en el sector metalmecánico y de alimentación (Rojas, 1995) permitió afirmar que las empresas eran renuentes a capacitar, $y$ que consideraban a la capacitación una inversión no redituable de manera inmediata. Según se pudo hipotetizar, los dirigentes se encontraban sujetos a mentalidades rentistas y no productivistas y existian prejuicios acerca de las capacidades genéticas de la fuerza de trabajo. En tal sentido, se observó la existencia de muy pocos programas de capacitación, no permanentes ni sistemáticos, ligados

12 Berrone Pascual. Evolución y perspectivas del sector lácteo. Bolsa de Comercio de Córdoba. Instituto de investigaciones económicas. Octubre de 1998. Pág. 23. 
más bien a los cambios tecnológicos, que mostraban un panorama de estancamiento en lo relativo a horas anuales de capacitación y cantidad de trabajadores capacitados.

El relevamiento emprendido en este estudio muestra una situación parcialmente distinta, aún cuando subsisten ciertos comportamientos que podrian asimilarse a los descriptos en el trabajo de investigación antes mencionado. El sector lácteo muestra una dinámica muy significativa en lo relativo a capacitación, pero esto no significa que la capacitación sea un valor de la cultura de empresa, ni que exista convicción en el empresariado acerca de su importancia, ni que se haya desligado de lo coyuntural -en este caso de los cambios tecnológicos y de la certificación de calidad-, ni que se considere "capacitables" a todos los trabajadores.

La idea de que la inversión en capacitación no retorna fácilmente porque el trabajador no aplica lo aprendido en el puesto de trabajo, de que es difícil retener la mano de obra capacitada, de que el personal con mucha antigüedad y escaso nivel educativo no es reconvertible mediante la capacitación y la concepción de que es necesario hacer capacitación "porque lo exigen las ISO" o "porque se puso una máquina nueva", son ideas frecuentes en la voz de los dirigentes del sector lácteo y operan de modo tal que la capacitación es vista -en muchos casos-, más como costo que como inversión, a la vez que la incorporación de pasantes y la priorización del nivel de educación en el ingreso de personal, resultan estrategias útiles para disminuir el supuesto "costo" de la capacitación.
Un dirigente de la EA, por ejemplo, señala que los mayores problemas en el personal se debieron a la incorporación de tecnología en manteca y en UAT: "nos equivocamos nosotros", sostiene. Afirma que para dotar de personal a esta nueva área de producción, decidieron utilizar gente que ya estaba trabajando en la fábrica, pensando que al tener alguna experiencia en la misma iba a resultar más fácil la capacitación y no fue así. Señala que en este caso hubiese sido más conveniente "incorporar gente nueva y empezar de cero, bajando costos".

Capacitadores y dirigentes de la ESIL, en cambio, señalan que estos problemas son en general responsabilidad de las empresas. Respecto de una empresa del medio afirmó uno de ellos:

"...tiene programas excelentes de capacitación, la parte de comunicación es muy buena, espectacular, pero no llega nunca a la base, a los operarios. Tenemos excelentes relaciones con esa empresa, pero trabajamos poco, alli la inversión que hacen no tiene retorno, y ése es un error muy grueso, porque el tema es que la capacitación no se articula con las necesidades de la gente, $y$ se hace muy lejos de las necesidades de la gente, muy lejos del puesto de trabajo, sin entrenamiento, sin motivación, sin estímulo..."

En sus estudios sobre competitividad estructural, Coriat (1998) afirma recurrentemente que la búsqueda permanente de reducción del costo laboral por parte de las empresas atenta contra la productividad en el mediano y largo plazo; que "no es con trabajo mal pago, poco calificado y poco formado como pueden obtenerse beneficios de productividad". 
Por el contrario, hay que incorporar trabajadores calificados, formarlos e invertir en organización. Según el autor, también la reducción de insumos está sujeta a las inversiones y "hace necesario gastar tres o cuatro veces más en formación, en organización, en comunicación y en logística de implantación de máquinas". La idea de productividad estructural que sostiene el autor, se afirma en la construcción de colectivos de savoir-faire a largo plazo, basados en una noción de saber amplia, que comprenda "los saberes en organización y en gestión de las interfases, los saberes de los colectivos asalariados."

En la exploración del sector lácteo, estas ideas de la relación costosproductividad no fueron las más frecuentes y en muchos casos aparecen en la voz de los dirigentes como política de la organización, pero luego en el estudio de casos fueron disconfirmadas en la versión que algunos trabajadores y dirigentes ofrecieron de la realidad cotidiana de las empresas.

\subsubsection{Las innovaciones tecnológicas}

Junto con la certificación de calidad, las innovaciones tecnológicas que realizan las empresas son un factor impulsor de acciones de capacitación. La información relevada muestra que existe una dinámica importante respecto de la innovación tecnológica. Las empresas afirman haber iniciado las innovaciones entre 1992 y 1994 y actualmente se reali- za algún cambio en ese sentido (ver Tabla 4), al menos una vez al año.

Las innovaciones cubren un amplio espectro: incorporación de una nueva máquina en un sector, instalación de nuevas líneas de producción, reestructuración de plantas e instalación de nuevas fábricas.

Excepto en el caso de la implantación de nuevas fábricas, en donde en general se contrata personal nuevo, las otras innovaciones impactan directamente en la capacitación. Se dijo antes, que una de las características de la capacitación del sector es que se sujeta con frecuencia a los cambios tecnológicos: la implementación de ISO y la innovación tecnológica son los dos factores que generan más acciones de capacitación en las empresas. En este marco, observamos que la capacitación sigue estando desvinculada del concepto de educación permanente, sin convertirse en una actividad integrada en todos los procesos. En muchos casos, la empresa apela a la capacitación cuando la máquina llegó, cuando la nueva tecnologia ya está funcionando, como un recurso mágico.

Aún en este marco general, existen diferencias entre la capacitación ligada a la certificación de calidad de la que surge de las innovaciones tecnológicas. En general, la capacitación ligada a ISO se desarrolla a través de programas que involucran a una masa crítica importante de trabajadores, mientras que la capacitación ligada a innovación tecnológica tiene alcances variables, de acuerdo a las dimensiones de la innovación. En muchos casos, cuando solo se cambian 


\section{Tabla 4}

Innovaciones tecnológicas realizadas por las empresas

\begin{tabular}{|c|c|c|}
\hline Empresas & Antigüedad & Tipo de Innovación \\
\hline A & Menos de un año & $\begin{array}{l}\text { Equipo nuevo para el sector de mantequería } \\
\text { y fraccionado de leche en polvo }\end{array}$ \\
\hline B & Entre tres y cinco años & Incorporación de planta de leche en polvo \\
\hline C & Menos de un año & Fábrica automática de quesos duros \\
\hline D & Menos de un año & $\begin{array}{l}\text { Dosificadora de yoghurt y sacheteadora } \\
\text { (máquina agrupadora de sachets en bandejas) }\end{array}$ \\
\hline $\mathbf{E}$ & Menos de un año & $\begin{array}{l}\text { Ampliación de planta de recepción y } \\
\text { procesamiento de materia prima }\end{array}$ \\
\hline $\mathrm{F}$ & Menos de un año & $\begin{array}{l}\text { Nueva línea para leche larga vida con } \\
\text { máquina esterilizadora }\end{array}$ \\
\hline G & Menos de un año & $\begin{array}{l}\text { Reestructuración de líneas de producción } \\
\text { en su planta más antigua }\end{array}$ \\
\hline
\end{tabular}

Fuente: Empresas encuestadas. Elaboración propia.

algunos componentes tecnológicos, es individual y personalizada. En este sentjdo, la capacitación centrada en la adquisición de nuevas habilidades en relación al uso de máquinas es muy considerada por los trabajadores y más aún cuando es individual o cuando acceder a ella otorga cierto prestigio.

En dos de las empresas (EA y EE) se instaló una máquina tetra pack (envases de cartón para leche o yoghurt) y se capacitó -de modo muy distinto en cada empresa-al personal. Si bien hubo simultáneamente varios cursos masivos de otra índole, ligados a calidad, la gente tiende a destacar la importancia de "saber operar máquinas", como la capacitación más valiosa.

EA: "Acá se busca que la gente que ingresa sepa operar las máquinas... la capacitación la recibimos en el puesto de trabajo, la gente que tiene más pasta enseña... trabaja en paralelo...; por ejemplo, en la tetra pack primero me pusieron directamente en el puesto con la máquina... después me mandaron a hacer el curso... eso lo hicieron para que no vaya tan crudo... después le enseñé yo a los operarios" (supervisor de envase)

EE: "Lo más importante es saber operar la máquina. A mí me mandaron a Estados Unidos a aprender a usar la tetra y cuando volví... bueno... yo soy el especialista en esto, no?" (supervisor de envase).

En lo relativo a las innovaciones es destacable la importancia que adquieren para los trabajadores los equipos, maquinarias y herramientas. Ya se trate de la incorporación de una máquina, de la renovación de una línea de producción o de la instalación de un nuevo sector, aún cuando los trabajadores no sean convocados por la empresa a participar en alguna instancia del proceso de innovación, suelen intervenir por propia iniciativa. 
En el caso de la calidad, cuatro de las siete empresas relevadas realizaron la implementación de normas en sus diferentes plantas utilizando mecanismos de participación e involucramiento destinados a integrar a los trabajadores al proceso, en distintas instancias y con distintos alcances, además de la participación en actividades de formación y capacitación sobre gestión de la calidad. En tres de ellas, se convocó a supervisores y operarios, que fueron involucrados en la determinación de los procedimientos, en la organización de círculos de calidad y en la discusión de criterios para el control preventivo. En la cuarta solo a operarios, especialmente en la fase de relevamiento de las ocupaciones.

Como se dijo, los trabajadores $-y$ muchos jefes y algunos gerentes-, se refieren al tema de la gestión de la calidad con cierta desconfianza e incredulidad. La mayoria reconocen la importancia de la gestión de calidad, comprenden la filosofía que implica e incluso se apropian parcialmente del discurso en tanto discurso dominante, pero pocos encuentran congruencia entre "o que se dice" y "o que se hace" en relación a este tema.

La información confirma que la gestión de la calidad es aceptada como innovación en la medida en que los discursos concuerden con las prácticas; cuando no es así, es percibida como algo ineficaz.

En este marco, cabe destacar que las expresiones de los trabajadores sobre este tema tienen un componente emocional importante: muchos no reconocen que les haya permitido producir mejoras en su desempeño personal o que hayan producido algún cambio significativo a nivel de las formas de trabajo y organización -a ex- cepción de dos casos, por la implementación de círculos de calidad y de la figura del lider de calidad-; mientras en muchos casos - a juicio de los supervisores o jefes- sí hubo cambios conductuales importantes en el desempeño del personal.

En lo relativo a los conocimientos, los trabajadores reconocen el impulso que las políticas de calidad han dado a la capacitación pero reclaman "que tenga continuidad", "que no solo se haga por certificar", así, es posible interpretar que las acciones de capacitación -aún cuando se desarrollen de modo aislado y coyuntural-, generan en los trabajadores expectativas de capacitación continua o permanente.

En el caso de las innovaciones en tecnologias "duras" la situación cambia sustantivamente. En primer término, se considera que las innovaciones en equipamientos, maquinarias $y$ herramientas son "concretas" y representan siempre algún cambio efectivo.

Como los procesos de innovación a gran escala suelen derivar en pérdida de puestos de trabajo, al inicio de estos procesos (1992, 1993 aproximadamente) las empresas experimentaron resistencia -explícita y/o implícita- por parte de los trabajadores; posteriormente, ya sea a través de algún mecanismo de inducción planificada o de modo espontáneo, el personal fue sumándose a los cambios, asumiendo incluso un rol protagónico.

En las entrevistas individuales $y$ grupales -tanto en las que fueron planificadas como en las ocasionales-, los trabajadores relatan procesos completos de innovación y se refieren específicamente a su importancia, al cambio que implica- 
ron y al papel que ellos mismos jugaron en dicho proceso:

"Yo también tuve que aprender esta elaboración específica... la ventaja es que yo entré a este lugar cuando todavía habla tierra... vi todo el montaje y participé en el mismo... cuando llegó la gente desde la Central, se encontró con todo armado y con una persona que sabía hacer todo... yo sabía cada uno de los pasos del proceso, sabía pasteurizar, hacer la manteca y fraccionar..."(supervisor de mantequería, EA).

"Usted no sabe lo que era hacer esto antes: todo a mano... todo lento. Por suerte cuando trajeron las máquinas nuevas, empezamos a trabajar dignamente... en realidad montamos todo nosotros... porque los ingenieros no interpretan bien la práctica y entonces uno ve que están por mandarse una macana e interviene. Por suerte no se enojan, al contrario, nos dejan participar en todo momento" (operador de envase, EE).

-Yo monté todo el laboratorio de queseria cuando vine para acá", se presenta ante el entrevistador un operador de quesería de la EA y relata a continuación el proceso de innovación desde su inicio.

- Todo esto (señala el sector desde una oficina vidriada en lo alto) lo instalamos nosotros. Los holandeses trajeron las máquinas un miércoles, las hicieron instalar junto con el servicio técnico un jueves, las probaron el viernes y se fueron el sábado. El lunes no podíamos hacer andar nada. Empezamos de cero con los muchachos. Incluso cambiamos máquinas de lugar y las pusimos a punto a nuestro modo. Al principio el Jefe se enojó, pero después, como veĺa que si nolas haciamos andar nosotros no las hacia andar nadie... aflojaron. Esto ha cambiado tantol! Le digo que son las máquinas más importantes del mundo, eh?" (supervisor de hojalatería, EG).

Actualmente las innovaciones son vistas como moneda corriente. Luego de ser comunicadas, sobreviene un breve período de incertidumbre y temor respecto de la posible pérdida de puestos, pero finalmente son bien recibidas, en tanto representan una mejora en las condiciones de trabajo y-como en el caso de casi todas las inversiones- una señal de estabilidad y/o crecimiento de la empresa, hoy sinónimo de mayores posibilidades de conservación de la fuente de trabajo.

Las acciones de capacitación derivadas de estas innovaciones son de tipo informal, -salvo el caso de equipos muy sofisticados-. Se suele capacitar a una o dos personas para que luego entrenen al resto del personal del sector. A su vez, la gente recibe muy bien el entrenamiento $e$ incluso busca "conocer" y "probar" casi todas las máquinas nuevas que estén a su alcance, lo que favorece la gestión de competencias de la empresa. Los trabajadores establecen una relación afectiva muy significativa con las máquinas y herramientas -en términos de "cuidarlas", "retarlas" o "felicitarlas" como si fueran personas-, a la vez que desarrollan al máximo las posibilidades instrumentales de las mismas asimilándolas al espacio vital del puesto de trabajo:

"...viste la máquina que está ahí, esa la maneja una persona que hace mucho que trabaja acá, le cambia de lugar el pie, lo pone del otro lado porque dice que como está no puede trabajar... se acostumbró asi y no puede hacerlo de otra forma... se demora tiempo en desarmar el 
pie y rearmarlo como él quiere, y eso perjudica la producción y significa perder dinero... pero bueno, la máquina es como una prolongación de uno mismo" (EA).

La naturaleza de la relación que une a los trabajadores a los equipamientos y maquinarias tecnológicas, contribuye a potenciar el carácter personalizado e informal que adquieren las actividades de capacitación que se emprenden como consecuencia de las innovaciones.

\section{Conclusiones}

- Un rasgo compartido por todo el grupo de empresas es que inician políticas y acciones de capacitación más o menos intensas a partir de 1992, en función de desafíos comerciales y productivos comunes: mejorar la calidad y la productividad, con vistas a una mejor competitividad.

- Las innovaciones tecnológicas y organizativas derivadas de estos desafíos y sus modos de gestión inciden significativamente en la capacitación y en la percepción que de ella tienen los actores. En este sentido, pudo observarse que la gestión inadecuada de las innovaciones y del clima social en que se desarrollan, pueden constituir factores limitantes de la socialización de saberes y producir fracturas importantes en la hetero-formación entre el personal de los distintos niveles y sectores de la empresa.

- Esto puede verse porque las estrategias de capacitación escogidas y las modalidades de implementación, no son necesariamente similares, aún cuando las empresas resulten similares en tamaño y complejidad, difieren sustantivamente según el origen de capital, la forma societal y la cultura organizacional derivada. Así, sus resultados son diversos y están más estrechamente vinculados a las condiciones socio-estructurales de cada empresa que a una lógica propia del sector productivo o del contexto más general.

- También puede afirmarse que los desafíos que entrentan las empresas para lograr competitividad, pueden ser considerados factores impulsores de acciones de capacitación, pero no constituyen bases para el desarrollo de políticas a largo plazo. En algunos procesos de reconversión, la formación y la capacitación logra instalarse como valor de la cultura; en otros, solo adquiere un carácter instrumental, coyuntural y contingente, siguiendo el ritmo y la importancia estratégica de las innovaciones. Algunas empresas, incluso, consideran a la capacitación más como un costo que como una inversión.

- Se observó que la gestión de las innovaciones producen un impacto importante en la división social del trabajo y en la composición social de las empresas: el proceso de reestructuración productiva ha dado origen a variaciones significativas en cuanto a la edad, el nivel educativo de ingreso y la calificación del personal: los cambios en el perfil de ingreso para el reclutamiento confirman la tendencia en las empresas a asegurarse una formación básica general y la existencia de los hábitos y disciplina, entendidos como indispensables para la producción. En la población ya inte- 
grada, se asiste a un fenómeno de homogeneización en términos de edad, educación formal y experiencia. La mayoría de la población actual (jefes, supervisores y operarios) son varones, de entre 31 y 45 años de edad, con formación de nivel medio completa, terciaria o universitaria incompleta y con experiencia en el oficio. El personal de más edad y antigüedad, constituye una minoría, frecuentemente desplazada de los procesos más automatizados.

\section{Bibliografía citada}

Aglietta, Michel (1979). Regulación y crisis del capitalismo. Siglo XXI. México.

Blanchet, Alain, Ghiglione, Rodolphe, Massonnat, Jean \& Trognon, Alain (1989). Técnicas de investigación en Ciencias Sociales. Editorial Narcea. Madrid.

Boyer, Robert (1989). La Teoria de la Regulación: un análisis critico. CEIL-Humanitas. Buenos Aires.

Catalano, Ana y Hemández, Daniel (1994). "(Re) Estructuración de la empresa y autonomía de los actores. Reflexión a partir de un estudio de caso" en Neffa, Julio (Comp) Nuevo paradigma productivo, flexibilidad y respuestas sindicales en América Latina. $1^{\circ}$ Reunión de la Red Franco-Latinoamericana "Trabajo y Tecnologias". Asociación Trabajo y Sociedad. Buenos Aires.

Coriat, Benjamin (1992), El taller y el robot. Ensayos sobre el fordismo y la producción en masa en la era de la electrónica. Siglo Veintiuno Editores. México.

Coriat, Benjamin (1998). Los desafios de la competitividad. Seminarios Intensivos de investigación. EUDEBA. Buenos Aires.
Gallart, Maria Antonia (1993). "La integración de métodos y la metodología cualitativa" en: Forni, Floreal H. Gallart, Maria Antonia \& Vasilachis de Gialdino, Irene Métodos cualitativos II. La práctica de la investigación. Centro Editor de América Latina. Buenos Aires.

Labarca, Guillermo (1999). Formación y empresa. Herramientas para la transformación. Centro Interamericano de investigación y Documentación sobre Formación Profesional (CINTERFOR). Montevideo.

Lipietz, Alain (1985). Mirages et miracles: Problémes de l'industrialisation dans le tiers monde. La Découverte. París.

Monteiro Leite, Elenice (1996). El rescate de la calificación. Herramientas para la transformación. Centro Interamericano de Investigación y Documentación sobre Formación Profesional (CINTERFOR). Montevideo.

Neffa, Julio (1998), Los paradigmas productivos taylorista y fordista y su crisis. Una contribución a su estudio desde la teoría de la regulación. Asociación Trabajo y Sociedad. Programa de Investigaciones Económicas sobre Tecnologia, Trabajo y Empleo PIETTE (CONICET). LumenHumanitas. Buenos Aires.

Novick, Marta y Catalano, Ana María (1996). "La Sociología del Trabajo al encuentro de las relaciones laborales en una marco de incertidumbre", en PANAIA, Marta (comp.) Trabajo y Empleo. Un abordaje interdisciplinario. Eudeba. Buenos Aires.

Novick, Marta y Gallart, María (1997). Competitividad, redes productivas y com* petencias laborales; ¿homogene. idad o segmentación? Herramientas para la transformación. Centro $\mathrm{ln}$ teramericano de Investigación y Documentación sobre Formación Profesional (CINTERFOR). Montevideo. 
Paín, Abraham (1996). Capacitación laboral: formación de Formadores. Ediciones Novedades Educativas. Facultad de Filosofia y Letras de la Universidad de Buenos Aires. Buenos Aires.

Rojas, Eduardo (1995). "La capacitación y la formación profesional", en Rojas, Eduardo, Catalano, Ana Maria, Hernández, Daniel, Rosendo, Ricardo \& Sladogna, Mónica Los sindicatos y la tecnologia: cambios técnicos y de organización en las industrias metalmecánica y de la alimentación en Argentina. Proyecto Regional Cambio Tecnológico y Mercado de Trabajo 14. Oficina Regional de la OIT para América Latina y el Caribe. Santiago de Chile.

Rojas, Eduardo (1999). El saber obrero y la innovación en la empresa. Herramientas para la transformación. Centro Interamericano de Investigación y Docu. mentación sobre Formación Profesional (CINTERFOR). Montevideo.
Ruffier, Jean (1998), La eficiencia productiva: cómo funcionan las fábricas. Herramientas para la transformación. Centro Interamericano de Investigación y Documentación sobre Formación Profesional (CINTERFOR), Montevideo.

Stankiewicz, François (1991). "Las estrategias de las empresas frente a los recursos humanos", en Stankiewicz, François (comp) Las estrategias de las empresas frente a los recursos huma* nos. Coediciones Humanitas - Programa Interdisciplinario de Investiga* ciones sobre Tecnología, Trabajo y Empleo (PROIITE). Buenos Aires.

Zarifian, Phillippe (1999). El modelo de competencia y los sistemas productivos. Papeles de la Oficina Técnica. Centro Interamericano de Investigación y Documentación sobre Formación Profesional (CINTERFOR). Montevideo. 